\title{
Adiposity and cancer at major anatomical sites: umbrella review of the literature
}

\author{
Maria Kyrgiou,,${ }^{1,2}$ Ilkka Kalliala, ${ }^{1}$ Georgios Markozannes, ${ }^{3}$ Marc J Gunter, ${ }^{4}$ Evangelos Paraskevaidis, ${ }^{5}$ \\ Hani Gabra, ${ }^{1,2}$ Pierre Martin-Hirsch,6,7 Konstantinos K Tsilidis ${ }^{3,8}$
}

${ }^{1}$ Department of Surgery and

Cancer, Institute of

Reproductive and

Developmental Biology, Faculty

of Medicine, Imperial College

London, London W12 ONN, UK

${ }^{2}$ West London Gynaecological

Cancer Centre, Queen

Charlotte's and Chelsea

Hospital, Hammersmith

Hospital, Imperial Healthcare

NHS Trust, London, UK

${ }^{3}$ Department of Hygiene and

Epidemiology, University of

loannina School of Medicine,

Ioannina, Greece

4 Section of Nutrition and

Metabolism, International

Agency for Research on Cancer, Lyon, France

${ }^{5}$ Department of Obstetrics and Gynaecology, University of

Ioannina, Ioannina, Greece

${ }^{6}$ Department of Gynaecologic

Oncology, Lancashire Teaching

Hospitals, Preston, UK

${ }^{7}$ Department of Biophysics,

University of Lancaster,

Lancaster, UK

${ }^{8}$ Department of Epidemiology and Biostatistics, School of

Public Health, Imperial College

London, London UK

Correspondence to: M Kyrgiou

m.kyrgiou@imperial.ac.uk

Additional material is published

online only. To view please visit

the journal online.

Cite this as: $B M J$ J 2017;356:j477

http://dx.doi.org/10.1136/bmj.j477

Accepted: 24 January 2017

\author{
ABSTRACT \\ OBJECTIVE \\ To evaluate the strength and validity of the evidence \\ for the association between adiposity and risk of \\ developing or dying from cancer. \\ DESIGN \\ Umbrella review of systematic reviews and meta- \\ analyses.
}

DATA SOURCES

PubMed, Embase, Cochrane Database of Systematic Reviews, and manual screening of retrieved references.

\section{ELIGIBILITY CRITERIA}

Systematic reviews or meta-analyses of observational studies that evaluated the association between indices of adiposity and risk of developing or dying from cancer.

\section{DATA SYNTHESIS}

Primary analysis focused on cohort studies exploring associations for continuous measures of adiposity.

The evidence was graded into strong, highly suggestive, suggestive, or weak after applying criteria that included the statistical significance of the random effects summary estimate and of the largest study in a meta-analysis, the number of cancer cases,

heterogeneity between studies, $95 \%$ prediction

intervals, small study effects, excess significance bias, and sensitivity analysis with credibility ceilings.

\section{RESULTS}

204 meta-analyses investigated associations between seven indices of adiposity and developing or dying from 36 primary cancers and their subtypes. Of the 95 meta-analyses that included cohort studies and used a continuous scale to measure adiposity, only 12 (13\%) associations for nine cancers were supported by strong evidence. An increase in body mass index was associated with a higher risk of developing oesophageal adenocarcinoma; colon and rectal cancer in men; biliary tract system and pancreatic cancer; endometrial cancer in premenopausal women; kidney cancer; and multiple myeloma. Weight gain and waist to hip circumference ratio were associated with higher risks of postmenopausal breast cancer in women who have never used hormone replacement therapy and endometrial cancer, respectively. The increase in the risk of developing cancer for every $5 \mathrm{~kg} / \mathrm{m}^{2}$ increase in body mass index ranged from $9 \%$ (relative risk 1.09 , $95 \%$ confidence interval 1.06 to 1.13 ) for rectal cancer among men to $56 \%(1.56,1.34$ to 1.81$)$ for biliary tract system cancer. The risk of postmenopausal breast cancer among women who have never used HRT increased by $11 \%$ for each $5 \mathrm{~kg}$ of weight gain in adulthood (1.11, 1.09 to 1.13), and the risk of endometrial cancer increased by $21 \%$ for each 0.1 increase in waist to hip ratio $(1.21,1.13$ to 1.29$)$. Five additional associations were supported by strong evidence when categorical measures of adiposity were included: weight gain with colorectal cancer; body mass index with gallbladder, gastric cardia, and ovarian cancer; and multiple myeloma mortality.

\section{CONCLUSIONS}

Although the association of adiposity with cancer risk has been extensively studied, associations for only 11 cancers (oesophageal adenocarcinoma, multiple myeloma, and cancers of the gastric cardia, colon, rectum, biliary tract system, pancreas, breast, endometrium, ovary, and kidney) were supported by strong evidence. Other associations could be genuine, but substantial uncertainty remains. Obesity is becoming one of the biggest problems in public health; evidence on the strength of the associated risks may allow finer selection of those at higher risk of cancer, who could be targeted for personalised prevention strategies.

\section{Introduction}

Cancer is a leading cause of death worldwide, with an estimated 12.7 million new cases and 7.6 million deaths from cancer annually. ${ }^{1}$ Excess body weight is associated with an increased risk of developing and dying from many diseases, including cancer, type 2 diabetes, and cardiovascular disease. ${ }^{2}$ Obesity has become a major public health challenge ${ }^{3}$; its prevalence worldwide has more than doubled among women and tripled among men in the past four decades. ${ }^{4}$ The number of overweight and obese people has risen from approximately 857 million in 1980 to 2.1 billion in 2013.5

Several meta-analyses support the link between obesity and cancer, but substantial heterogeneity exists 
between studies. ${ }^{6}$ The reported associations may be causal, but they may also be flawed, as inherent study biases such as residual confounding and selective reporting of positive results may exaggerate the effect of obesity on cancer. ${ }^{7-10}$ A recent umbrella review found that, despite strong claims of a statistically significant association between type 2 diabetes and several cancers, only a fraction (14\%) of the 27 studied associations were supported by robust evidence, without any potential bias. ${ }^{11}$

To summarise and evaluate the existing evidence and appraise its quality, we performed an umbrella review of systematic reviews and meta-analyses that investigated the association between adiposity indices and risk of developing or dying from cancer. Umbrella reviews systematically appraise the evidence on an entire topic across many meta-analyses of multiple putative risk factors on multiple outcomes. ${ }^{11-18}$

\section{Methods \\ Literature search}

We searched PubMed, Embase, and the Cochrane Database of Systematic Reviews from inception to May 2015 for systematic reviews or meta-analyses that investigated the association between adiposity indices and risk of developing or dying from any cancer using a predefined search algorithm (see the supplementary file for details of the algorithm). Adiposity indices were body mass index, waist circumference, hip circumference, waist to hip ratio, weight, weight gain, and weight loss from bariatric surgery. We hand searched the references of the retrieved systematic reviews and meta-analyses and the proceedings of relevant conferences for articles missed by the electronic search and unpublished data.

\section{Inclusion and exclusion criteria}

We included systematic reviews and meta-analyses of observational epidemiological studies in humans that assessed continuous or categorical measures of adiposity. We excluded studies in which physical activity, height, or prenatal factors (such as birth weight or maternal obesity during pregnancy) were the exposures of interest and those in which cancer incidence or mortality were not the outcomes of interest. We excluded meta-analyses of prognostic studies that investigated cancer survival or other outcomes among patients with cancer and meta-analyses that did not include comprehensive data from individual studies-such as relative risks, 95\% confidence intervals, number of cases and controls, or total population. When we found more than one meta-analysis on the same association between exposure and outcome, we included the one with the largest number of primary studies to avoid duplication. We subsequently examined whether the summary estimates showed concordance between the included and duplicate meta-analyses.

\section{Data extraction}

We extracted the name of the first author, year of publication, outcome, and exposure from each eligible systematic review or meta-analysis. From each individual study in a meta-analysis, we extracted the first author, year of publication, epidemiological design, number of cases and controls in case-control studies or total population in cohort studies, maximally adjusted relative risk (odds ratio in case-control studies and hazard ratio or standardised incidence or mortality ratio in cohort studies) and 95\% confidence intervals. Two investigators (IK, MK) independently searched the literature, assessed the eligibility of the retrieved papers, and extracted the data. Disagreements were resolved by discussion with a third investigator (KT).

\section{Data analysis}

Estimation of summary effect-For each exposure and outcome pair, we calculated the summary effect and the 95\% confidence interval using fixed and random effects methods. ${ }^{19}$

Assessment of heterogeneity-The heterogeneity between studies was assessed with Cochran's Q test ${ }^{20}$ and the $\mathrm{I}^{2}$ statistic. ${ }^{21}$ Substantial inconsistency could reflect either genuine heterogeneity between studies or bias. To assess the uncertainty around heterogeneity estimates, we calculated 95\% confidence intervals. ${ }^{22}$

Estimation of prediction intervals-To further account for heterogeneity between studies we calculated 95\% prediction intervals for the summary random effect estimates, which represent the range in which the effect estimates of future studies will lie. ${ }^{23}$

Assessment of small study effects-We examined whether smaller studies gave higher risk estimates than larger studies, which is an indication of publication bias, true heterogeneity, or chance. ${ }^{1124}$ Indication of small study effects was based on the Egger's regression asymmetry test $(\mathrm{P} \leq 0.10)$ and whether the random effects summary estimate was larger than the point estimate of the largest study in the meta-analysis.

Evaluation of excess significance-We assessed excess significance bias by evaluating whether the observed number of studies with nominally statistically significant results ("positive" studies, $\mathrm{P}<0.05$ ) in the published literature was different from the expected number of studies with statistically significant results. ${ }^{25}$ The expected number of statistically significant studies in each meta-analysis was calculated from the sum of the statistical power estimates for each component study using an algorithm from a non-central $t$ distribution. ${ }^{2627}$ The power estimates of each component study depend on the plausible effect size for the tested association, which was assumed to be the effect of the largest study (that is, the smallest standard error) in each meta-analysis. ${ }^{28}$ Sensitivity analyses were conducted using the summary fixed and random effects estimates as alternative plausible effect sizes. Excess significance for individual meta-analyses was determined at $\mathrm{P} \leq 0.10 .^{25}$

Credibility ceilings-We used credibility ceilings, a sensitivity analysis tool, to account for potential methodological limitations of observational studies that might lead to spurious precision of combined effect estimates. ${ }^{29}$ The main assumption of this method is that 
every observational study has a probability $c$ (credibility ceiling) that the true effect size is in a different direction from the one suggested by the point estimate. The pooled effect size and the heterogeneity between studies were re-estimated using a wide range of credibility ceiling values. ${ }^{2930}$

\section{Grading the evidence}

The associations between measures of adiposity and cancer were categorised into strong, highly suggestive, suggestive, or weak depending on the strength and validity of the evidence. ${ }^{131518}$ A strong association was claimed when the $\mathrm{P}$ value of the random effects meta-analysis was smaller than $10^{-6}$, a threshold that might substantially reduce false positive findings ${ }^{31-33}$ if the meta-analysis has more than 1000 cancer cases, the largest study in the meta-analysis is nominally statistically significant $(\mathrm{P}<0.05)$, the $\mathrm{I}^{2}$ statistic of heterogeneity between studies is smaller than $50 \%$, the $95 \%$ prediction intervals excludes the null value, small study effects or excess significance bias are not indicated, and the association survives a credibility ceiling of at least $10 \%$.

The association was considered highly suggestive if $\mathrm{P}<10^{-6}$, the meta-analysis had more than 1000 cases, and the largest study in the meta-analysis was nominally statistically significant. The criteria for suggestive were: random effects $\mathrm{P}<10^{-3}$ and the meta-analysis had more than 1000 cases. All other associations with a nominally statistically significant $P$ value for the random effects meta-analysis were deemed to provide weak evidence.

The primary analysis in this umbrella review focused on cohort studies using continuous measures of adiposity, which was considered a more valid and standardised way of presenting and synthesising individual study estimates than using categorical measures. Sensitivity analyses were conducted using case-control studies and categorical measures. All statistical analyses were performed using Stata version 13 (College Station, TX), ${ }^{34}$ and all $\mathrm{P}$ values were two tailed.

\section{Patient involvement}

No patients were involved in setting the research question or outcome measures, nor were they involved in developing plans for design or implementation of the study. No patients were asked to advise on interpretation or writing up on results. The results will be disseminated to the general public through public presentations and the authors' involvement in different charities.

\section{Results}

Characteristics of the meta-analyses

Overall, 49 eligible papers were identified that included a total of 204 meta-analyses. These meta-analyses summarised 2179 individual study estimates from 507 unique cohort or case-control studies (fig 1). ${ }^{35-83}$ Of the 507 unique studies, 371 (73.2\%) had a cohort design, 134 (26.4\%) were case-control studies, and two (0.4\%) were cross sectional studies. A median of 7 (range 2-72) study

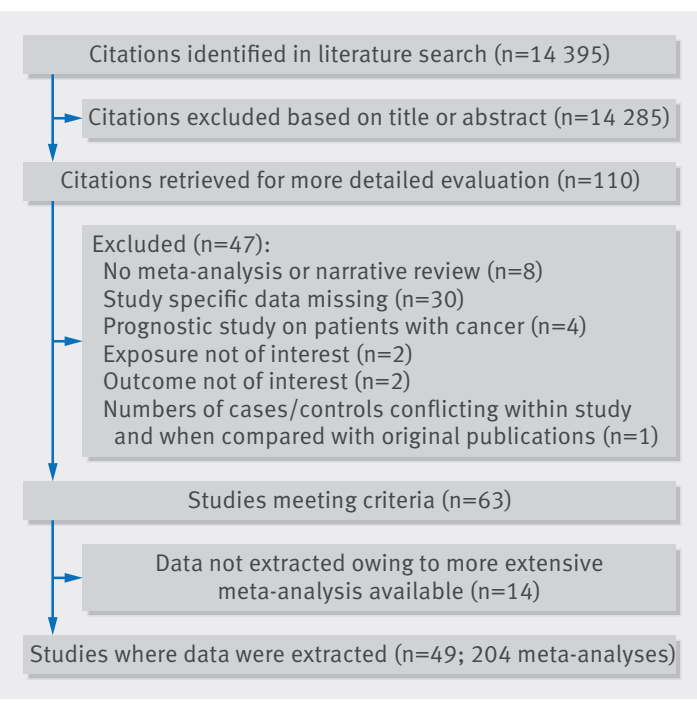

Fig 1 | Study flowchart

estimates were combined for each meta-analysis. The median number of cases and population or controls in each meta-analysis was 5645 and 1766389 , respectively. A total of 177 meta-analyses had at least 1000 cancer cases.

The 204 meta-analyses included associations between seven indices of adiposity (body mass index, waist circumference, hip circumference, waist to hip ratio, weight, weight gain, and weight loss through bariatric surgery) and the incidence $(n=196)$ or mortality $(n=8)$ from cancer at 36 anatomical sites and by subtypes. A total of 194 meta-analyses included cohort studies (see supplementary tables 1 and 2). Of these, 95 used a continuous scale to measure adiposity (for example, body mass index in adults or at around age 20 per $5 \mathrm{~kg} / \mathrm{m}^{2}$, waist circumference per $10 \mathrm{~cm}$, hip circumference per $10 \mathrm{~cm}$, waist to hip ratio per 0.1, weight per $5 \mathrm{~kg}$, and weight gain per $1 \mathrm{~kg}$ or $5 \mathrm{~kg}$ ). These 95 meta-analyses studied 28 different cancer sites or subtypes and included 818 individual study estimates, with body mass index in adults being investigated most frequently ( $\mathrm{n}=57$ meta-analyses).

We identified more than one published meta-analysis with the same exposure and outcome pair for 11 cancers. All duplicate meta-analyses showed agreement on the direction, magnitude, and statistical significance of the summary associations (see supplementary table 3).

\section{Summary effect size}

When we used a threshold of $\mathrm{P}<0.05$, the summary fixed effects estimates were significant in 76 of the 95 meta-analyses (80\%) and the summary random effects were significant in 72 (76\%) (see supplementary table 1 and supplementary figures 1-28). At a stricter threshold of $\mathrm{P}<0.001,66$ (69\%) and 59 (62\%) meta-analyses produced significant summary results using the fixed and random effects models, respectively. At $\mathrm{P}<10^{-6}, 54$ (57\%) and 35 (37\%) meta-analyses were significant, respectively.

Of those 35 meta-analyses, 31 found an increased risk of cancer with higher adiposity for oesophageal adenocarcinoma, multiple myeloma, and cancers of the 


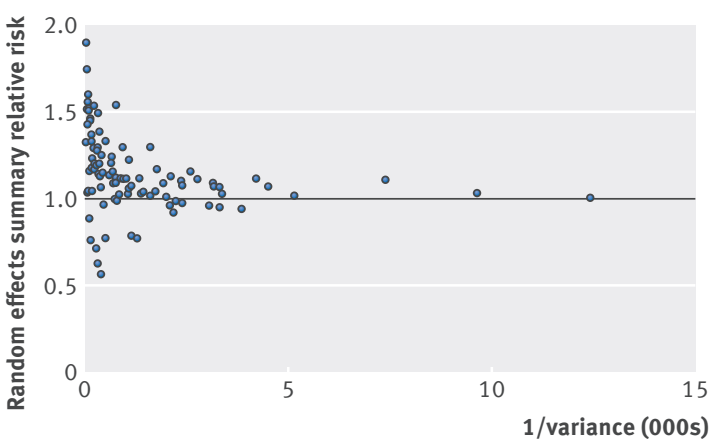

Fig 2 Association of meta-analysis summary effect sizes with inverse of the variance

colon, rectum, liver, biliary tract system (cancers of gallbladder, extrahepatic bile duct, and ampulla of Vater), pancreas, postmenopausal breast, endometrium, and kidney. Four of the 35 meta-analyses indicated an inverse association for oesophageal squamous cell carcinoma and lung cancer. The magnitude of the observed summary random effect estimates ranged from 0.57 to $1.90 ; 61 \%$ of the estimates lay between 0.80 and 1.20 (fig 2). In meta-analyses with small variances a trend of summary effects towards 1.00 was observed. The largest study of each meta-analysis showed a nominally statistically significant association in 65 meta-analyses (68\%), and the relative risks of the largest studies were more conservative (closer to null) than the summary random effects in $53(56 \%)$ meta-analyses.

The summary estimates were similar between men and women for eight of 11 cancers (oesophageal adenocarcinoma, oesophageal squamous cell carcinoma, multiple myeloma, leukaemia, and gastric, lung, kidney, and thyroid cancers). However, the incidence of colon and rectal cancer was higher per $5 \mathrm{~kg} / \mathrm{m}^{2}$ increase of body mass index in men (relative risk 1.30, 95\% confidence interval 1.25 to 1.35) and 1.09 (1.06 to 1.13), respectively) than in women (1.12 (1.06 to 1.17) and 1.02 (0.99 to 1.05), respectively). This was also true for other adiposity indices. Moreover, body mass index was associated with an increased risk of melanoma in men (relative risk per $5 \mathrm{~kg} / \mathrm{m}^{2} 1.17,1.05$ to 1.30 ) but not in women (0.96, 0.93 to 1.00$)$.

\section{Heterogeneity between studies}

The $\mathrm{Q}$ test was significant at $\mathrm{P} \leq 0.10$ in 42 of 95 meta-analyses (44\%) (see supplementary table 2). Twenty meta-analyses (21\%) showed considerable heterogeneity $\left(\mathrm{I}^{2}=50-75 \%\right)$ and 15 meta-analyses $(16 \%)$ showed substantial heterogeneity $\left(\mathrm{I}^{2}>75 \%\right.$ ) for some adiposity indices and several cancers (oesophageal squamous cell carcinoma; colon, liver, lung, endometrial, prostate, and thyroid cancers; melanoma; and leukaemia). We further assessed the uncertainty of the summary effects by calculating $95 \%$ prediction intervals; the null value was excluded in only 27 associations.

\section{Small study effects}

Thirteen meta-analyses had evidence indicating small study effects, but only seven included 10 or more studies and therefore had enough statistical power for the Egger's test to adequately identify presence of small study effects (colon, liver, lung, ovarian, and advanced prostate cancer; see supplementary table 2). Supplementary figures 1 to 28 show the funnel plots for each cancer site.

\section{Excess significance}

Nineteen meta-analyses had evidence of excess significance bias for some adiposity indices using the largest study estimate as the plausible effect size (colon, rectal, liver, pancreatic, endometrial, ovarian, prostate, advanced prostate, and kidney cancer; supplementary table 2). Using summary fixed or random effects estimates as plausible effect sizes gave similar results.

\section{Credibility ceilings}

A credibility ceiling of $0 \%$ corresponds to the random effects model calculation. Of the 95 meta-analyses, 72 $(76 \%)$ retained nominal statistical significance $(\mathrm{P}<0.05)$ with a credibility ceiling of 5\%. Fifty (53\%), 33 (35\%), and $19(20 \%)$ meta-analyses remained statistically significant with ceilings of $10 \%, 15 \%$, and $20 \%$, respectively. Heterogeneity between studies gradually decreased with increasing ceilings. With a ceiling of $10 \%$, no meta-analyses of cohort studies with continuous exposure gave an $\mathrm{I}^{2}$ estimate larger than 50\% (supplementary table 4).

\section{Grading the evidence}

We explored whether the reported associations between adiposity and developing or dying from cancer were supported by strong, highly suggestive, suggestive, or weak evidence (table 1). We found that $77 \%$ of the 95 meta-analyses presented at least weak evidence $(\mathrm{P}<0.05$ for the summary random effects). Supplementary table 5 shows these associations in cohort studies using continuous measures of adiposity (also see supplementary table 6 for associations using categorical measures of adiposity in cohort studies and supplementary table 7 for all associations regardless of study type or exposure metric).

Only 13\% of meta-analyses (12 of 95) were supported by strong evidence. They summarised data on body mass index $(n=10)$, waist to hip ratio $(n=1)$, and weight gain $(n=1)$. An increase in body mass index was associated with a higher risk of developing oesophageal adenocarcinoma; colon and rectal cancer in men; biliary tract system and pancreatic cancer; endometrial cancer in premenopausal women; kidney cancer; and multiple myeloma. Weight gain and waist to hip circumference ratio were associated with higher risks of postmenopausal breast cancer in women who have never used hormone replacement therapy and endometrial cancer, respectively. The increase in the risk of developing cancer for every $5 \mathrm{~kg} / \mathrm{m}^{2}$ increase in body mass index ranged from 9\% (relative risk 1.09, 95\% confidence interval 1.06 to 1.13) for colorectal cancer among men to $56 \%(1.56,1.34$ to 1.81$)$ for biliary tract system cancer. The risk of postmenopausal breast cancer among women who have never used HRT increased by $11 \%$ for 


\begin{tabular}{|c|c|c|c|}
\hline Evidence & Criteria used & Decreased risk & Increased risk \\
\hline $\begin{array}{l}\text { Strong } \\
(\mathrm{n}=12)\end{array}$ & $\begin{array}{l}P<10^{-6 *} ;>1000 \text { cases; } P<0.05 \text { of } \\
\text { largest study in meta-analysis; }\left.\right|^{2} \\
<50 \% \text {; no small study effectt; } \\
\text { prediction interval excludes null } \\
\text { value; no excess significance biasł; } \\
\text { survive } 10 \% \text { credibility ceiling }\end{array}$ & None & $\begin{array}{l}\text { Oesophageal adenocarincoma (BMI); colon cancer, men (BMI); rectal cancer, men } \\
\text { (BMI); biliary tract system cancer§ (BMI); pancreatic cancer (BMI); postmenopausal } \\
\text { breast cancer, never HRT use (WG); endometrial cancer (WHR); premenopausal } \\
\text { endometrial cancer (BMI); kidney cancer, men and women (BMI); multiple myeloma, } \\
\text { overall and women (BMI) }\end{array}$ \\
\hline $\begin{array}{l}\text { Highly } \\
\text { suggestive } \\
(n=17)\end{array}$ & $\begin{array}{l}\mathrm{P}<10^{-6 *} ;>1000 \text { cases; } \mathrm{P}<0.05 \text { of } \\
\text { largest study in meta-analysis }\end{array}$ & $\begin{array}{l}\text { Oesophageal squamous cell } \\
\text { carcinoma, overall and women } \\
\text { (BMI); lung cancer, overall and men } \\
\text { (BMI) }\end{array}$ & $\begin{array}{l}\text { Colon cancer (BMI and waist circumference per } 10 \mathrm{~cm} \text { ); liver cancer (BMI); } \\
\text { postmenopausal breast cancer (BMI); endometrial cancer (BMI, BMI in young } \\
\text { adulthood, weight per } 5 \mathrm{~kg} \text {, WG); postmenopausal endometrial cancer (BMI); } \\
\text { endometrial cancer, type I (BMI); endometrial cancer, type II (BMI); kidney cancer (BMI) }\end{array}$ \\
\hline $\begin{array}{l}\text { Suggestive } \\
(n=23)\end{array}$ & $P<10^{-3 *} ;>1000$ cases & $\begin{array}{l}\text { Oesophageal squamous cell } \\
\text { carcinoma, men (BMI); lung cancer, } \\
\text { smokers (BMI); premenopausal } \\
\text { breast cancer (BMI); localised } \\
\text { prostate cancer (BMI) }\end{array}$ & $\begin{array}{l}\text { Colon cancer, women (BMI); colon cancer, men and overall (WG); colon cancer (WHR } \\
\text { and WC); colorectal cancer (WG per } 1 \mathrm{~kg}) \text {; rectal cancer (BMI); pancreatic cancer } \\
\text { (WHR and WC); ovarian cancer (BMI and BMI in young adulthood); prostate cancer } \\
\text { mortality (BMI); thyroid cancer, overall and women (BMI); non-Hodgkin's lymphoma } \\
\text { (BMI); multiple myeloma, men (BMI); leukaemia (BMI) }\end{array}$ \\
\hline $\begin{array}{l}\text { Weak } \\
(n=19)\end{array}$ & $P<0.05^{\star}$ & $\begin{array}{l}\text { Lung cancer, women (BMI); } \\
\text { melanoma, women (BMI) }\end{array}$ & $\begin{array}{l}\text { Oesophageal adenocarcinoma in men and women (BMI); melanoma, men (BMI); } \\
\text { endometrial cancer (HC per } 10 \mathrm{~cm}) \text {; postmenopausal endometrial cancer, never HRT } \\
\text { use (BMI and WG); postmenopausal endometrial cancer, ever HRT use (BMI and WG); } \\
\text { endometrial cancer mortality (BMI); ovarian cancer (weight per } 5 \mathrm{~kg} \text { ); } \\
\text { postmenopausal ovarian cancer, never HRT use (WG); prostate cancer, advanced } \\
\text { (BMI); prostate cancer, countries with high screening rate for prostate specific } \\
\text { antigen (WG); thyroid cancer, men (BMI); non-Hodgkin's lymphoma mortality (BMI); } \\
\text { leukaemia, men and women (BMI) }\end{array}$ \\
\hline \multicolumn{4}{|c|}{$\begin{array}{l}\left.\left.\text { BMI=body mass index (per } 5 \mathrm{~kg} / \mathrm{m}^{2}\right) ; \mathrm{HC}=\text { hip circumference; } \mathrm{HRT}=\text { hormone replacement therapy; WHR=waist to hip ratio (per } 0.1 \text { units); WC=waist circumference (per } 10 \mathrm{~cm}\right) \text {; WG=weight gain } \\
\text { (per } 5 \mathrm{~kg} \text { unless otherwise stated) } \\
\text { *P value of meta-analysis random effects model. } \\
\text { †Small study effect is based on P value from Egger's regression asymmetry test (P>0.1) where the random effects summary estimate was larger compared to the point estimate of the largest } \\
\text { study in a meta-analysis. } \\
\text { fBased on P>0.1 of excess significance test using largest study (smallest standard error) in meta-analysis as plausible effect size. } \\
\text { §Includes cancers of gallbladder, extrahepatic bile duct, and ampulla of Vater. }\end{array}$} \\
\hline
\end{tabular}

each $5 \mathrm{~kg}$ of weight gain $(1.11,1.09$ to1.13), and the risk of endometrial cancer increased by $21 \%$ for each 0.1 increase in waist to hip ratio $(1.21,1.13$ to 1.29$)$.

Seventeen meta-analyses (18\%) were supported by highly suggestive evidence, and they found positive associations for colon (with body mass index and waist circumference), liver (with body mass index), postmenopausal breast (with body mass index), total endometrial (with body mass index in adults and at around age 20, waist circumference, weight, and weight gain), postmenopausal, type I and type II endometrial (with body mass index), and kidney cancer (with body mass index). Inverse associations were found for body mass index in adults with oesophageal squamous cell carcinoma (overall and in women) and lung cancer (overall and in men). Twenty four meta-analyses (25\%) were supported by suggestive evidence for an association, and 19 meta-analyses (20\%) were supported by weak evidence.

The evidence was graded similarly in the 194 meta-analyses of cohort studies that assessed continuous and categorical measures of adiposity (supplementary table 6). Five additional associations were supported by strong evidence: weight gain with incidence of colorectal cancer, body mass index with incidence of gallbladder, gastric cardia, and ovarian cancers, and mortality from multiple myeloma. When both cohort and case-control studies were assessed for continuous and categorical measures of adiposity (supplementary table 7), two additional associations were supported by strong evidence: body mass index and risk of melanoma and meningioma, both of which were judged as weak associations when only cohort studies were evaluated. The strong evidence for an association between body mass index and incidence of biliary tract system cancerwas downgraded to suggestive evidence when both case-control and cohort studies were evaluated.

\section{Discussion}

We reviewed 204 meta-analyses to evaluate the current evidence for associations between seven adiposity indices and the risk of developing or dying from 36 primary cancers and their subtypes. Twelve associations, stemming from cohort studies using continuous measures of adiposity, were supported by strong evidence, inferred by strongly statistically significant results and no suggestion of bias. These associations were primarily between body mass index and malignancies of digestive organs (oesophageal adenocarcinoma and cancers of the colorectum (in men only), biliary tract system, and pancreas), hormone related cancers (such as postmenopausal breast in women who have never used HRT), premenopausal and overall endometrial cancer, kidney cancer, and multiple myeloma. Five additional associations were supported by strong evidence when categorical measures of adiposity were used: weight gain with risk of colorectal cancer risk and body mass index with risk of gallbladder, gastric cardia, and ovarian cancer, and mortality from multiple myeloma.

The effect of obesity on the incidence and mortality of cancer is well recognised 66284 and was evident in our umbrella review, with approximately $77 \%$ of the included meta-analyses reporting a nominally statistically significant summary random effects estimate. Although the reported associations are plausibly 
accurate and potentially causal, the risk of reporting, selection, and other inherent biases may overestimate the suggested associations, as shown in other recently published umbrella reviews in cancer epidemiology. ${ }^{11} 1327$ The extent to which the literature is affected by such biases is difficult to prove definitively. We used statistical tests and sensitivity analyses to look for evidence of bias. When lower $\mathrm{P}$ value thresholds $\left(\mathrm{P}<10^{-6}\right)$ were used, the proportion of significant associations decreased to $37 \%$. Large heterogeneity $\left(\mathrm{I}^{2} \geq 50 \%\right)$ was observed in $37 \%$ of the meta-analyses. When we calculated the $95 \%$ prediction intervals, which further account for heterogeneity, we found that the null value was excluded in only about one third of the associations. Moreover, some meta-analyses had evidence of small study effects or excess significance bias. Most meta-analyses (53\%) preserved their statistical significance with a $10 \%$ credibility ceiling, but only one in five preserved significance at a ceiling of $20 \%$, indicating that many associations between adiposity and cancer remain uncertain.

\section{Comparison with other studies}

Our grading of the evidence largely agrees with systematic analyses of the literature performed by the World Cancer Research Fund (WCRF) and the International Agency for Research on Cancer (IARC). ${ }^{6485}$ The WCRF currently lists seven cancers for which the evidence supports a convincing causal relationship with obesity (oesophageal adenocarcinoma and cancers of the pancreas, colorectum, postmenopausal breast, endometrium, kidney, and liver). We also found strong evidence that obesity increases the risk of these cancers, except for liver cancer, for which the evidence was considered highly suggestive because of small study effects, excess significance bias, and substantial heterogeneity between studies. The association between obesity and five more malignancies (gallbladder, stomach cardia, ovarian, advanced prostate, and premenopausal breast cancer) was graded as probably causal by WCRF and received lower evidence grades in our main analysis using only continuous measures of adiposity. IARC found sufficient evidence to support the association between excess body fat and 13 of 24 cancer sites (oesophagus (adenocarcinoma), gastric cardia, colorectum, liver, gallbladder, pancreas, postmenopausal breast, endometrium, ovary, kidney, meningioma, thyroid, and multiple myeloma). ${ }^{84}$ Our results are similar to those of the IARC report for most cancers, except for gastric cardia, liver, ovarian, meningioma and thyroid cancers. These cancers received lower than strong evidence grades in our main analysis, owing to small numbers of cancer cases, very large heterogeneity between studies, or evidence of small study effects and excess significance bias. However, the associations between adiposity and risk of gastric cardia and ovarian cancer were judged to be supported with strong evidence when we evaluated categorical measures of adiposity. Similarly, evidence for an association between adiposity and risk of meningioma was considered strong when case-control studies were included in the evaluation.
Several methods exist for rating evidence, but they are inconsistent and allow some degree of arbitrariness. ${ }^{86}$ The WCRF and IARC used expert groups and criteria similar to Bradford Hill criteria to assess the association between obesity and risk of 17 and 24 primary cancers, respectively, whereas we assessed the robustness of the evidence using sensitivity analyses and statistical tests to evaluate 36 primary cancers and their subtypes, defined by anatomical location, histology, and receptor status. We also explored associations by potential effect modifiers (eg, sex, menopausal status, smoking status, and use of HRT). Our criteria for grading evidence should not be considered causal criteria, especially when used individually, but we think that they are useful for identifying biases when used together. ${ }^{12}$

Most of the associations between adiposity indices and endometrial cancer were supported by strong or highly suggestive evidence. In particular, the association between waist to hip ratio and risk of total endometrial cancer was supported by strong evidence, indicating that central obesity, which is linked to hyperinsulinaemia and type 2 diabetes, has a major role in the development of this disease. Strong evidence also supported the association between body mass index and premenopausal endometrial cancer. We found the associations between body mass index in adults or at around age 20, waist circumference, weight, and weight gain and total endometrial cancer, and between body mass index and postmenopausal type I and II endometrial cancer to be highly suggestive owing to substantial heterogeneity between studies and potential for excess significance bias. These results were in agreement with the WCRF and IARC, which did not provide separate ratings for menopausal status or histological subtype of disease. ${ }^{8487}$ The difference in evidence ratings between premenopausal and postmenopausal endometrial cancer is probably related to heterogeneity caused by use of HRT among postmenopausal women. ${ }^{88}$ We found that the associations between body mass index or weight gain and postmenopausal endometrial cancer were statistically significantly stronger in never users compared with ever users of HRT, but the relevant meta-analyses included only 2-6 studies and had fewer than 1000 cancer cases, so the evidence was deemed weak. The WCRF found that the associations between waist circumference or waist to hip ratio and total endometrial cancer were probably causal, ${ }^{87}$ which largely agreed with our findings.

We found strong evidence to support the association between weight gain in adulthood and postmenopausal breast cancer among women who have never used HRT, yielding an $11 \%$ higher risk per $5 \mathrm{~kg}$ of weight gain. We found highly suggestive evidence to support the positive association of body mass index with postmenopausal breast cancer, irrespective of HRT. Weight gain may be a better metric than body mass index for measuring the dynamic nature of adiposity during adulthood, when obesity becomes central and has more metabolic effects. ${ }^{49}$ Another reason for the lower grading of the evidence for body mass index was the large 
heterogeneity between studies, ${ }^{62}$ which could at least partially be explained by a potential interaction with HRT use. Some cohort studies have found a positive association between body mass index and postmenopausal breast cancer only among women who have never used HRT, 8990 but we did not capture this potential interaction because published meta-analyses have not performed this subgroup analysis. ${ }^{6278}$ The evidence for an association between adiposity indices and premenopausal breast cancer was weaker and only reached suggestive evidence for an inverse association with body mass index in a meta-analysis of 20 cohort studies. These results largely agree with the WCRF and IARC findings of a convincing causal positive association between adiposity and postmenopausal breast cancer ${ }^{8491}$ and a probable causal inverse association for premenopausal disease. ${ }^{91}$ Two recent Mendelian randomisation studies found that a higher body mass index in adults was associated with a lower risk of postmenopausal breast cancer, contradicting the epidemiological evidence. ${ }^{9293}$ This may be due to the genetic risk score for body mass index in adults being a stronger determinant of early compared with later life body mass index; epidemiological studies have found inverse associations between body mass index in childhood and both premenopausal and postmenopausal breast cancer. ${ }^{9495}$ The positive association between body mass index in adults and postmenopausal breast cancer found in epidemiological studies may be driven by weight gain in adulthood. We found strong evidence to support the association between weight gain in adults and postmenopausal breast cancer, but weight gain is probably linked to environmental factors that are not captured by genetic risk factors. ${ }^{96}$ Moreover, some evidence exists that these associations may differ according to oestrogen and progesterone receptor status of the tumour (supplementary tables 6 and 7), ${ }^{689}$ but large investigations in this area are lacking.

The association between body mass index and colon cancer was supported by strong evidence in men and suggestive evidence in women. Twenty four cohorts investigated the association in men, totalling a 30\% higher risk of colon cancer for each $5 \mathrm{~kg} / \mathrm{m}^{2}$ increase in body mass index. The risk was 12\% higher for each $5 \mathrm{~kg} /$ $\mathrm{m}^{2}$ increase in body mass index among women in a meta-analysis of 20 cohorts that showed substantial heterogeneity and evidence of small study effects and excess significance bias. The association between body mass index and rectal cancer in men was supported by strong evidence, but with a summary relative risk considerably smaller than for colon cancer $(1.09 v 1.30)$. We found no association between body mass index and rectal cancer in women. The evidence for most of the other adiposity indices was suggestive, as few studies have investigated these associations. The insulin signalling pathway is a possible mechanism underlying the association between obesity and colorectal cancer in men.98 The increased concentrations of circulating insulin induced by adiposity are higher among men than women, ${ }^{99}$ and men are more prone to abdominal fatness than women. ${ }^{100}$ Furthermore, endogenous and exogenous oestrogens have been associated with protective effects against colorectal cancer among women, which might explain why the association of adiposity with colorectal cancer is stronger in men than in women. ${ }^{101} 102$ Two Mendelian randomisation studies found statistically significant positive associations between adult body mass index and colorectal cancer but did not perform analyses by sex. ${ }^{92} 103$

We found that higher body mass index was associated with an increased risk of oesophageal adenocarcinoma, and this was supported by strong evidence. We found an inverse association for oesophageal squamous cell carcinoma, which was supported by highly suggestive evidence due to considerable heterogeneity between studies. Similar summary associations were found by the WCRF and IARC, which concluded that adiposity convincingly increased the risk of oesophageal adenocarcinoma. ${ }^{84104}$ A Mendelian randomisation study supports this association. ${ }^{105}$ Oesophageal squamous cell carcinoma has stronger associations with smoking and alcohol consumption than oesophageal adenocarcinoma, ${ }^{106}$ but we were unable to capture the potential residual confounding or effect modification of smoking and alcohol in the associations between adiposity and oesophageal squamous cell carcinoma because the published meta-analysis did not report these subgroup analyses. ${ }^{6}$

We found an inverse association between body mass index and lung cancer that was supported by highly suggestive evidence but had substantial heterogeneity between studies and evidence of small study effects. When we investigated this association by smoking status, we found an inverse association between body mass index and lung cancer among smokers, which was supported by suggestive evidence. We found a non-significant association among non-smokers. These results may be due to residual confounding, reverse causation, or effect modification by smoking, which led the WCRF and IARC to grade this evidence as inadequate. ${ }^{84107}$ Two recent Mendelian randomisation studies found that body mass index was statistically significantly associated with an increased risk of squamous and small cell lung cancer but had a borderline significant inverse association with lung adenocarcinoma. ${ }^{92108}$ One of the Mendelian randomisation studies found a statistically significant inverse association between body mass index and total lung cancer among never smokers and a null association in ever smokers, ${ }^{108}$ which contradicts the epidemiological evidence. However, these analyses were underpowered. Future large prospective studies should evaluate associations according to smoking status among different disease subtypes.

Furthermore, we found that the associations between body mass index and multiple myeloma and cancers of the biliary tract system, pancreas, and kidney were supported by strong evidence. The WCRF and IARC ratings were similar, except the WCRF did not study multiple myeloma. ${ }^{84} 85$ The associations for less common malignancies are supported by limited data and show substantial heterogeneity between studies; we need prospective studies to better characterise these associations. 
Limitations of this study

Our review relied on previously published meta-analyses and the literature searches performed by the authors of those meta-analyses. Some studies may have been missed, although this is unlikely to have influenced our findings because our assessment of duplicate meta-analyses on the same exposure-outcome associations gave similar summary results. Some of the meta-analyses that used continuous measures of adiposity were published as long ago as 2008, which may mean that we didn't include the most recent evidence. However, we found that the evidence grading for recently published meta-analyses investigating the association of categorical terms of body mass index with the same cancers was similar.

We evaluated all study specific results that were reported in the meta-analyses (for example, primary cancers, cancer subtypes, sex, menopausal status, smoking status, and HRT use), but we may have missed other subanalyses that were not reported with sufficient study specific data. Assessing the quality of the primary studies included in the meta-analyses was beyond the scope of this umbrella review. Finally, the statistical tests we used to assess bias do not prove its definitive presence or its exact source. However, our estimates are likely to be conservative, as a negative test does not exclude the potential for bias. Moreover, our evidence grading was not sensitive to the use of $95 \%$ prediction intervals, excess significance tests, or credibility ceilings, because when we consecutively removed these criteria from the quality of evidence algorithm, the resulting inference remained the same.

\section{Conclusion}

The association between obesity and risk of developing or dying from cancer has been extensively studied. We found strong evidence to support the positive association between obesity and 11 of the 36 cancer sites and subtypes that we examined, predominantly comprising cancers of the digestive organs and hormone related malignancies in women. Substantial uncertainty remains for the other cancers. To draw firmer conclusions we need prospective studies and large consortiums with better assessment of the changing nature of body fatness and with comprehensive standardised reporting of analyses. As obesity becomes one of the greatest public health problems worldwide, evidence of the strength of the associations between obesity and cancer may allow finer selection of people at high risk, who could be selected for personalised primary and secondary prevention strategies.

Contributors: MK, PMH, EP, and KKT conceived and designed the study. IK, MK, and KT acquired and collated the data. IK, MK, GM, and KKT analysed the data. All authors drafted and critically revised the manuscript for important intellectual content and gave final approval of the version to be published. MK and IK contributed equally to the manuscript (joint first authors). MK is the guarantor.

Funding: This work was supported by Genesis Research Trust (Garfield Weston Foundation, P63522 to MK); Sigrid Jusélius Fellowship (P52483 to IK and MK); World Cancer Research Fund International Regular Grant Programme (2014/1180 to KKT); Ovarian Cancer Action (PS5827 and PSA601 to MG and MK); the Imperial Experimental Cancer Medicine Centre, the Cancer Research UK Imperial Centre, Imperial
Healthcare NHS Trust NIHR BRC (P45272:MK). None of the funders had any influence on the study design; in the collection, analysis, and interpretation of data; in the writing of the report; and in the decision to submit the article for publication.

Competing interests: All authors have completed the ICMJE uniform disclosure form at www.icmje.org/coi disclosure.pdf and declare: no support from any organisation for the submitted work; no financial relationships with any organisations that might have an interest in the submitted work in the previous three years; no other relationships or activities that could appear to have influenced the submitted work. Ethical approval: Not required.

Data sharing: No additional data available.

Transparency: The lead author (MK) affirms that the manuscript is an honest, accurate, and transparent account of the study being reported; that no important aspects of the study have been omitted; and that any discrepancies from the study as planned have been explained.

This is an Open Access article distributed in accordance with the terms of the Creative Commons Attribution (CC BY 4.0) license, which permits others to distribute, remix, adapt and build upon this work, for commercial use, provided the original work is properly cited. See: http://creativecommons.org/licenses/by/4.0/

1 Ferlay J, Shin HR, Bray F, Forman D, Mathers C, Parkin DM. Estimates of worldwide burden of cancer in 2008: GLOBOCAN 2008. Int / Cancer 2010;127:2893-917. doi:10.1002/ijc.25516.

Dixon JB. The effect of obesity on health outcomes. Mol Cell Endocrinol 2010;316:104-8. doi:10.1016/j.mce.2009.07.008. 3 Stevens GA, Singh GM, Lu Y, et al. Global Burden of Metabolic Risk Factors of Chronic Diseases Collaborating Group (Body Mass Index). National, regional, and global trends in adult overweight and obesity prevalences. Popul Health Metr 2012;10:22. doi:10.1186/1478-7954-10-22

4 NCD Risk Factor Collaboration (NCD-RisC). Trends in adult body-mass index in 200 countries from 1975 to 2014: a pooled analysis of 1698 population-based measurement studies with $19 \bullet 2$ million participants. Lancet 2016:387:1377-96. doi:10.1016/ S0140-6736(16)30054-X

$5 \mathrm{Ng}$ M, Fleming T, Robinson M, et al. Global, regional, and national prevalence of overweight and obesity in children and adults during 1980-2013: a systematic analysis for the Global Burden of Disease Study 2013. Lancet 2014;384:766-81. doi:10.1016/ S0140-6736(14)60460-8.

6 Marmot M, Atinmo T, Byers T, et al. Food, nutrition, physical activity, and the prevention of cancer: a global perspective. World Cancer Research Fund/ American Institute for Cancer Research, 2007

7 Ioannidis JP. Why most discovered true associations are inflated. Epidemiology 2008;19:640-8. doi:10.1097/EDE.0b013e31818131e7.

8 loannidis JP. Why most published research findings are false. PLOS Med 2005;2:e124. doi:10.1371/journal.pmed.0020124.

9 Dwan K, Gamble C, Williamson PR, Kirkham JJ, Reporting Bias G. Reporting Bias Group. Systematic review of the empirical evidence of study publication bias and outcome reporting bias - an updated review. PLoS One 2013;8:e66844. doi:10.1371/journal. pone.0066844.

10 Boffetta P, McLaughlin JK, La Vecchia C, Tarone RE, Lipworth L, Blot W. False-positive results in cancer epidemiology: a plea for epistemological modesty. J Natl Cancer Inst 2008;100:988-95. doi:10.1093/jnci/djn191

11 Tsilidis KK, Kasimis JC, Lopez DS, Ntzani EE, Ioannidis JP. Type 2 diabetes and cancer: umbrella review of meta-analyses of observational studies. BMJ 2015;350:g7607. doi:10.1136/bmj.g7607.

12 Ioannidis JP. Integration of evidence from multiple meta-analyses: a primer on umbrella reviews, treatment networks and multiple treatments meta-analyses. CMAJ 2009;181:488-93. doi:10.1503/ cmaj.081086

13 Markozannes G, Tzoulaki I, Karli D, et al. Diet, body size, physical activity and risk of prostate cancer: An umbrella review of the evidence. Eur J Cancer 2016;69:61-9. doi:10.1016/j.ejca.2016.09.026.

14 Belbasis L, Savvidou MD, Kanu C, Evangelou E, Tzoulaki I. Birth weight in relation to health and disease in later life: an umbrella review of systematic reviews and meta-analyses. BMC Med 2016;14:147. doi:10.1186/s12916-016-0692-5.

15 Bellou V, Belbasis L, Tzoulaki I, Evangelou E, Ioannidis JP. Environmental risk factors and Parkinson's disease: An umbrella review of meta-analyses. Parkinsonism Relat Disord 2016;23:1-9. doi:10.1016/j.parkreldis.2015.12.008.

16 Belbasis L, Bellou V, Evangelou E, Ioannidis JP, Tzoulaki . Environmental risk factors and multiple sclerosis: an umbrella review of systematic reviews and meta-analyses. Lancet Neurol 2015;14:263 73. doi:10.1016/S1474-4422(14)70267-4.

17 Theodoratou E, Tzoulaki I, Zgaga L, Ioannidis JP. Vitamin D and multiple health outcomes: umbrella review of systematic reviews and meta-analyses of observational studies and randomised trials. BMJ 2014:348:g2035. doi:10.1136/bmj.g2035. 
18 Bellou V, Belbasis L, Tzoulaki I, Middleton LT, Ioannidis JP, Evangelou E. Systematic evaluation of the associations between environmental risk factors and dementia: An umbrella review of systematic reviews and meta-analyses. Alzheimers Dement 2016;S1552-5260(16)32853-9.

19 DerSimonian R, Laird N. Meta-analysis in clinical trials. Control Clin Trials 1986;7:177-88. doi:10.1016/0197-2456(86)90046-2.

20 Cochran WG. The combination of estimates from different experiments. Biometrics 1954;10:101-29doi:10.2307/3001666.

21 Higgins JP, Thompson SG, Deeks JJ, Altman DG. Measuring inconsistency in meta-analyses. BM/ 2003:327:557-60. doi:10.1136/ bmj.327.7414.557.

22 Ioannidis JP, Patsopoulos NA, Evangelou E. Uncertainty in heterogeneity estimates in meta-analyses. BMJ 2007;335:914-6. doi:10.1136/bmj.39343.408449.80.

23 Riley RD, Higgins JP, Deeks JJ. Interpretation of random effects meta-analyses. BM/ 2011:342:d549. doi:10.1136/bmj.d549.

24 Sterne JA, Sutton AJ, loannidis JP, et al. Recommendations for examining and interpreting funnel plot asymmetry in meta-analyses of randomised controlled trials. BMJ 2011;343:d4002. doi:10.1136/ bmi.d4002.

25 Ioannidis JP, Trikalinos TA. An exploratory test for an excess of significant findings. Clin Trials 2007:4:245-53. doi:10.1177/1740774507079441.

26 Tsilidis KK, Panagiotou OA, Sena ES, et al. Evaluation of excess significance bias in animal studies of neurological diseases. PLoS Biol 2013;11:e1001609. doi:10.1371/journal.pbio.1001609.

27 Tsilidis KK, Papatheodorou SI, Evangelou E, Ioannidis JP. Evaluation of excess statistical significance in meta-analyses of 98 biomarker associations with cancer risk. J Natl Cancer Inst 2012;104:1867-78. doi:10.1093/jnci/djs437.

28 Ioannidis JPA. Clarifications on the application and interpretation of the test for excess significance and its extensions. J Math Psychol 2013;57:184-7doi:10.1016/i.jmp.2013.03.002.

29 Salanti G, loannidis JP. Synthesis of observational studies should consider credibility ceilings. J Clin Epidemiol 2009;62:115-22. doi:10.1016/j.jclinepi.2008.05.014.

30 Papatheodorou SI, Tsilidis KK, Evangelou E, Ioannidis JP. Application of credibility ceilings probes the robustness of meta-analyses of biomarkers and cancer risk. J Clin Epidemiol 2015;68:163-74. doi:10.1016/j.jclinepi.2014.09.004

31 Ioannidis JP, Tarone R, McLaughlin JK. The false-positive to false-negative ratio in epidemiologic studies. Epidemiology 2011;22:450-6. doi:10.1097/EDE.0b013e31821b506e.

32 Sterne JA, Davey Smith G. Sifting the evidence-what's wrong with significance tests?BMJ 2001;322:226-31. doi:10.1136/ bmj.322.7280.226.

33 Johnson VE. Revised standards for statistical evidence. Proc Natl Acad SciU S A 2013:110:19313-7. doi:10.1073/pnas.1313476110.

34 StataCorp. Stata Statistical Software: Release 13.StataCorp LP, 2013.

35 Alsamarrai A, Das SL, Windsor JA, Petrov MS. Factors that affect risk for pancreatic disease in the general population: a systematic review and meta-analysis of prospective cohort studies. Clin Gastroenterol Hepatol 2014;12:1635-44.e5, quiz e103. doi:10.1016/j. cgh.2014.01.038.

36 Amadou A, Ferrari P, Muwonge R, et al. Overweight, obesity and risk of premenopausal breast cancer according to ethnicity: a systematic review and dose-response meta-analysis. Obes Rev 2013;14:665-78. doi:10.1111/obr.12028.

37 Aune D, Greenwood DC, Chan DS, et al. Body mass index, abdominal fatness and pancreatic cancer risk: a systematic review and non-linear dose-response meta-analysis of prospective studies. Ann Oncol 2012:23:843-52 doi:10.1093/annonc/mdr398.

38 Aune D, Navarro Rosenblatt DA, Chan DS, et al. Anthropometric factors and ovarian cancer risk: a systematic review and nonlinear dose-response meta-analysis of prospective studies. Int J Cancer 2015;136:1888-98. doi:10.1002/ijc.29207.

39 Aune D, Navarro Rosenblatt DA, Chan DS, et al. Anthropometric factors and endometrial cancer risk: a systematic review and dose-response meta-analysis of prospective studies. Ann Oncol 2015;26:1635-48. doi:10.1093/annonc/mdv142.

40 Cao Y, Ma J. Body mass index, prostate cancer-specific mortality, and biochemical recurrence: a systematic review and meta-analysis. CancerPrev Res (Phila) 2011;4:486-501. doi:10.1158/1940-6207. CAPR-10-0229.

41 Casagrande DS, Rosa DD, Umpierre D, Sarmento RA, Rodrigues CG, Schaan BD. Incidence of cancer following bariatric surgery: systematic review and meta-analysis. Obes Surg 2014;24:1499-509. doi:10.1007/s11695-014-1276-0.

42 Castillo JJ, Ingham RR, Reagan JL, Furman M, Dalia S, Mitri J. Obesity is associated with increased relative risk of diffuse large B-cell lymphoma: a meta-analysis of observational studies. Clin Lymphoma Myeloma Leuk 2014;14:122-30. doi:10.1016/j.clml.2013.10.005.

43 Castillo JJ, Reagan JL, Ingham RR, et al. Obesity but not overweight increases the incidence and mortality of leukemia in adults: a meta-analysis of prospective cohort studies. Leuk Res 2012;36:86875. doi:10.1016/j.leukres.2011.12.020.
44 Chen Y, Wang X, Wang J, Yan Z, Luo J. Excess body weight and the risk of primary liver cancer: an updated meta-analysis of prospective studies. Eur / Cancer 2012;48:2137-45. doi:10.1016/j. ejca.2012.02.063

45 Connolly BS, Barnett C, Vogt KN, Li T, Stone J, Boyd NF. A meta-analysis of published literature on waist-to-hip ratio and risk of breast cancer. Nutr Cancer 2002;44:127-38 doi:10.1207/S15327914NC4402 02

46 Discacciati A, Orsini N, Wolk A. Body mass index and incidence of localized and advanced prostate cancer--a dose-response meta-analysis of prospective studies. Ann Oncol 2012;23:1665-71. doi:10.1093/annonc/mdr603.

47 Harriss DJ, Atkinson G, George K, et al. C-CLEAR group. Lifestyle factors and colorectal cancer risk (1): systematic review and meta-analysis of associations with body mass index. Colorectal Dis 2009;11:547-63. doi:10.1111/j.1463-1318.2009.01766.x

48 Harvie M, Hooper L, Howell AH. Central obesity and breast cancer risk: a systematic review. Obes Rev 2003:4:157-73. doi:10.1046/j.1467-789X.2003.00108.x.

49 Keum N, Greenwood DC, Lee DH, et al. Adult weight gain and adiposity-related cancers: a dose-response meta-analysis of prospective observational studies. I Natl Cancer Inst 2015;107doi:10.1093/jnci/djv088

50 Larsson SC, Wolk A. Obesity and colon and rectal cancer risk: a meta-analysis of prospective studies. Am I Clin Nutr 2007;86:556-65.

51 Larsson SC, Wolk A. Obesity and risk of non-Hodgkin's lymphoma: a meta-analysis. Int J Cancer 2007;121:1564-70. doi:10.1002/ijc.22762.

52 Larsson SC, Wolk A. Obesity and the risk of gallbladder cancer: a meta-analysis. BrJ Cancer 2007;96:1457-61.

53 Larsson SC, Wolk A. Body mass index and risk of non-Hodgkin's and Hodgkin's lymphoma: a meta-analysis of prospective studies. Eur J Cancer 2011;47:2422-30. doi:10.1016/j.ejca.2011.06.029.

54 Li JS, Han TJ, Jing N, et al. Obesity and the risk of cholangiocarcinoma: a meta-analysis. Tumour Biol 2014;35:6831-8. doi:10.1007/ s13277-014-1939-4.

55 Lin XJ, Wang CP, Liu XD, et al. Body mass index and risk of gastric cancer: a meta-analysis. Jpn J Clin Oncol 2014;44:783-91. doi:10.1093/jico/hyu082

56 Ma J, Huang M, Wang L, Ye W, Tong Y, Wang H. Obesity and risk of thyroid cancer: evidence from a meta-analysis of 21 observational studies. Med Sci Monit 2015;21:283-91. doi:10.12659/MSM.892035.

57 Ma Y, Yang Y, Wang F, et al. Obesity and risk of colorectal cancer: systematic review of prospective studies. PLoS One 2013;8:e53916. doi:10.1371/journal.pone.0053916.

58 MacInnis RJ, English DR. Body size and composition and prostate cancer risk: systematic review and meta-regression analysis. Cancer Causes Control 2006;17:989-1003. doi:10.1007/s10552-006-0049-z.

59 Moghaddam AA, Woodward M, Huxley R. Obesity and risk of colorectal cancer: a meta-analysis of 31 studies with 70,000 events. Cancer Epidemiol Biomarkers Prev 2007;16:2533-47. doi:10.1158/1055-9965.EPI-07-0708

60 Park M, Song DY, Je Y, Lee JE. Body mass index and biliary tract disease: a systematic review and meta-analysis of prospective studies. Prev Med 2014;65:13-22. doi:10.1016/j.ypmed.2014.03.027.

61 Poorolajal J, Jenabi E. The association between BMI and cervical cancer risk: a meta-analysis. Eur / Cancer Prev 2016;25:232-8. doi:10.1097/CEJ.0000000000000164.

62 Renehan AG, Tyson M, Egger M, Heller RF, Zwahlen M. Body-mass index and incidence of cancer: a systematic review and meta-analysis of prospective observational studies. Lancet 2008;371:569-78. doi:10.1016/S0140-6736(08)60269-X

63 Schlesinger S, Lieb W, Koch M, et al. Body weight gain and risk of colorectal cancer: a systematic review and meta-analysis of observational studies. Obes Rev 2015;16:607-19. doi:10.1111/ obr.12286.

64 Sergentanis TN, Antoniadis AG, Gogas HJ, et al. Obesity and risk of malignant melanoma: a meta-analysis of cohort and case-control studies. Eur J Cancer 2013;49:642-57. doi:10.1016/j. ejca.2012.08.028

65 Shao C, Bai LP, Qi ZY, Hui GZ, Wang Z. Overweight, obesity and meningioma risk: a meta-analysis. PLoS One 2014;9:e90167. doi:10.1371/journal.pone.0090167.

66 Singh S, Sharma AN, Murad MH, et al. Central adiposity is associated with increased risk of esophageal inflammation, metaplasia, and adenocarcinoma: a systematic review and meta-analysis. Clin Gastroenterol Hepatol 2013;11:1399-1412.e7. doi:10.1016/j. cgh.2013.05.009.

67 Sun JW, Zhao LG, Yang Y, Ma X, Wang YY, Xiang YB. Obesity and risk of bladder cancer: a dose-response meta-analysis of 15 cohort studies. PLoS One 2015;10:e0119313. doi:10.1371/journal.pone.0119313.

68 Suzuki R, Orsini N, Saji S, Key TJ, Wolk A. Body weight and incidence of breast cancer defined by estrogen and progesterone receptor status--a meta-analysis. Int / Cancer 2009;124:698-712. doi:10.1002/ ijc. 23943

69 Upala S, Anawin S. Bariatric surgery and risk of postoperative endometrial cancer: a systematic review and meta-analysis. Surg Obes Relat Dis 2015;11:949-55. doi:10.1016/j.soard.2014.09.024. 
70 Wallin A, Larsson SC. Body mass index and risk of multiple myeloma: a meta-analysis of prospective studies. Eur J Cancer 2011;47:1606-15. doi:10.1016/j.ejca.2011.01.020.

71 Wang F, Xu Y. Body mass index and risk of renal cell cancer: a dose-response meta-analysis of published cohort studies. Int J Cancer 2014:135:1673-86. doi:10.1002/ijc.28813.

72 Wang Y, Wang B, Shen F, Fan J, Cao H. Body mass index and risk of primary liver cancer: a meta-analysis of prospective studies. Oncologist 2012:17:1461-8. doi:10.1634/theoncologist.2012-0066.

73 Yang XW, Li PZ, Zhu LY, Zhu S. Effects of bariatric surgery on incidence of obesity-related cancers: a meta-analysis. Med Sci Monit 2015;21:1350-7. doi:10.12659/MSM.893553.

74 Yang Y, Dong J, Sun K, et al. Obesity and incidence of lung cancer: a meta-analysis. Int J Cancer 2013;132:1162-9. doi:10.1002/ijc.27719.

75 Zhang X, Zhou G, Sun B, et al. Impact of obesity upon prostate cancer-associated mortality: A meta-analysis of 17 cohort studies. Oncol Lett 2015:9:1307-12

76 Zhang Y, Liu H, Yang S, Zhang J, Qian L, Chen X. Overweight, obesity and endometrial cancer risk: results from a systematic review and meta-analysis. Int I Biol Markers 2014:29:e21-9. doi:10.5301/ jbm.5000047.

77 Poorolajal J, Jenabi E, Masoumi SZ. Body mass index effects on risk of ovarian cancer: a meta- analysis. Asian Pac J Cancer Prev 2014:15:7665-71. doi:10.7314/APICP.2014.15.18.7665.

78 Cheraghi Z, Poorolajal J, Hashem T, Esmailnasab N, Doosti Irani A. Effect of body mass index on breast cancer during premenopausal and postmenopausal periods: a meta-analysis. PLoS One 2012;7:e51446. doi:10.1371/journal.pone.0051446

79 Crosbie EJ, Zwahlen M, Kitchener HC, Egger M, Renehan AG. Body mass index, hormone replacement therapy, and endometrial cancer risk: a meta-analysis. Cancer Epidemiol Biomarkers Prev 2010;19:3119-30. doi:10.1158/1055-9965.EPI-10-0832.

80 Robsahm TE, Aagnes B, Hjartåker A, Langseth H, Bray Fl, Larsen IK. Body mass index, physical activity, and colorectal cancer by anatomical subsites: a systematic review and meta-analysis of cohort studies. Eur J Cancer Prev 2013;22:492-505. doi:10.1097/ CEJ.0b013e328360f434.

81 Turati F, Tramacere I, La Vecchia C, Negri E. A meta-analysis of body mass index and esophageal and gastric cardia adenocarcinoma. Ann Oncol 2013;24:609-17. doi:10.1093/annonc/mds244.

82 Larsson SC, WolkA. Overweight and obesity and incidence of leukemia: a meta-analysis of cohort studies. Int J Cancer 2008;122:1418-21. doi:10.1002/ijc.23176.

83 Pierobon M, Frankenfeld CL. Obesity as a risk factor for triple-negative breast cancers: a systematic review and meta-analysis. Breast Cancer Res Treat 2013;137:307-14. doi:10.1007/s10549-012-2339-3.

84 Lauby-Secretan B, Scoccianti C, Loomis D, Grosse Y, Bianchini F, Straif K. International Agency for Research on Cancer Handbook Working Group. Body Fatness and Cancer--Viewpoint of the IARC Working Group. N Engl J Med 2016;375:794-8. doi:10.1056/NEJMsr1606602.

85 World Cancer Research Fund CUP. http://www.wcrf.org/int/ research-we-fund/continuous-update-project-cup.

86 Guyatt GH, Oxman AD, Vist GE, et al. GRADE Working Group. GRADE: an emerging consensus on rating quality of evidence and strength of recommendations. BMJ 2008;336:924-6. doi:10.1136/ bmj.39489.470347.AD

87 World Cancer Research Fund International/American Institute for Cancer Research Continuous Update Project Report. Diet, Nutrition, Physical Activity, and Endometrial Cancer. 2013; Available at: http:// www.wcrf.org/sites/default/files/Endometrial-Cancer-2013-Report.pdf.

88 McCullough ML, Patel AV, Patel R, et al. Body mass and endometrial cancer risk by hormone replacement therapy and cancer subtype. Cancer Epidemiol Biomarkers Prev 2008;17:73-9. doi:10.1158/10559965.EPI-07-2567.

89 Lahmann PH, Hoffmann K, Allen N, et al. Body size and breast cancer risk: findings from the European Prospective Investigation into Cancer And Nutrition (EPIC). Int J Cancer 2004;111:762-71. doi:10.1002/ ijc.20315.

90 White KK, Park SY, Kolonel LN, Henderson BE, Wilkens LR. Body size and breast cancer risk: the Multiethnic Cohort. Int J Cancer 2012;131:E705-16. doi:10.1002/ijc.27373.
91 World Cancer Research Fund International/American Institute for Cancer Research Continuous Update Project Report. Diet, Nutrition, Physical Activity, and Breast Cancer. 2010; Available at: http://www. wcrf.org/sites/default/files/Breast-Cancer-2010-Report.pdf.

92 Gao C, Patel CJ, Michailidou K, et al. on behalf of: the Colorectal Transdisciplinary Study (CORECT); Discovery, Biology and Risk of Inherited Variants in Breast Cancer (DRIVE); Elucidating Loci Involved in Prostate Cancer Susceptibility (ELLIPSE); Follow-up of Ovarian Cancer Genetic Association and Interaction Studies (FOCI); and Transdisciplinary Research in Cancer of the Lung (TRICL). Mendelian randomization study of adiposity-related traits and risk of breast, ovarian, prostate, lung and colorectal cancer. Int J Epidemiol 2016:45:896-908. doi:10.1093/ije/dyw129.

93 Guo Y, Warren Andersen S, Shu XO, et al. Genetically Predicted Body Mass Index and Breast Cancer Risk: Mendelian Randomization Analyses of Data from 145,000 Women of European Descent. PLoS Med 2016:13:e1002105. doi:10.1371/journal.pmed.1002105.

94 Baer HJ, Tworoger SS, Hankinson SE, Willett WC. Body fatness at young ages and risk of breast cancer throughout life. Am J Epidemiol 2010;171:1183-94. doi:10.1093/aje/kwq045.

95 Baer HJ, Colditz GA, Rosner B, et al. Body fatness during childhood and adolescence and incidence of breast cancer in premenopausal women: a prospective cohort study. Breast Cancer Res 2005;7:R31425. doi:10.1186/bcr998.

96 Sandholt CH, Allin KH, Toft U, et al. The effect of GWAS identified BMI loci on changes in body weight among middle-aged Danes during a five-year period. Obesity (Silver Spring) 2014;22:901-8. doi:10.1002/ oby. 20540 .

97 Ritte R, Lukanova A, Berrino F, et al. Adiposity, hormone replacement therapy use and breast cancer risk by age and hormone receptor status: a large prospective cohort study. Breast Cancer Res 2012;14:R76. doi:10.1186/bcr3186.

98 Renehan AG, Zwahlen M, Minder C, O’Dwyer ST, Shalet SM, Egger M. Insulin-like growth factor (IGF)-I, IGF binding protein-3, and cancer risk: systematic review and meta-regression analysis. Lancet 2004;363:1346-53. doi:10.1016/S0140-6736(04)16044-3.

99 Kaaks R, Lukanova A, Kurzer MS. Obesity, endogenous hormones, and endometrial cancer risk: a synthetic review. Cancer Epidemiol Biomarkers Prev 2002;11:1531-43.

100 Geer EB, Shen W. Gender differences in insulin resistance, body composition, and energy balance. Gend Med 2009;6(Suppl 1):60-75. doi:10.1016/j.genm.2009.02.002

101 Murphy N, Strickler HD, Stanczyk FZ, et al. A Prospective Evaluation of Endogenous Sex Hormone Levels and Colorectal Cancer Risk in Postmenopausal Women. J Natl Cancer Inst 2015;107:djv210. doi:10.1093/jnci/djv210.

102 Chlebowski RT, Wactawski-Wende J, Ritenbaugh C, et al. Women's Health Initiative Investigators. Estrogen plus progestin and colorectal cancer in postmenopausal women. N Engl J Med 2004;350:991-1004. doi:10.1056/NEJMoa032071.

103 Thrift AP, Gong J, Peters U, et al. Mendelian Randomization Study of Body Mass Index and Colorectal Cancer Risk. Cancer Epidemiol Biomarkers Prev 2015;24:1024-31. doi:10.1158/1055-9965.EPI-14-1309.

104 World Cancer Research Fund International/American Institute for Cancer Research CUPR. Diet, Nutrition, Physical Activity, and Oesophageal Cancer. 2016; Available at: http://www.wcrf.org/sites/ default/files/Oesophageal-Cancer-2016-Report.pdf.

105 Thrift AP, Shaheen NJ, Gammon MD, et al. Obesity and risk of esophageal adenocarcinoma and Barrett's esophagus: a Mendelian randomization study. J Natl Cancer Inst 2014;106:dju252. doi:10.1093/inci/dju252.

106 Freedman ND, Abnet CC, Leitzmann MF, et al. A prospective study of tobacco, alcohol, and the risk of esophageal and gastric cancer subtypes. Am J Epidemiol 2007;165:1424-33. doi:10.1093/aje/kwm051.

107 World Cancer Research Fund International/American Institute for Cancer Research. Food, Nutrition, Physical Activity, and the Prevention of Cancer: A Global Perspective.AICR, 2007.

108 Carreras-Torres R, Haycock PC, Relton CL, et al. The causal relevance of body mass index in different histological types of lung cancer: A Mendelian randomization study. Sci Rep 2016;6:31121. doi:10.1038/ srep31121.

Supplementary figures

Supplementary tables and methods 